\title{
Roles of ZIC family genes in human gastric cancer
}

\author{
GANG MA $^{1}$, WEIJIE DAI ${ }^{1}$, AIYU SANG ${ }^{2}$, XIAOZHONG YANG $^{1}$ and QIANJUN LI ${ }^{1}$ \\ ${ }^{1}$ Department of Gastroenterology, Huai'an First People's Hospital, Nanjing Medical University, Huai'an, Jiangsu 223300; \\ ${ }^{2}$ Department of Internal Medicine, Lianshui Third People's Hospital, Lianshui, Jiangsu 223411, P.R. China
}

Received October 20, 2015; Accepted April 25, 2016

DOI: $10.3892 /$ ijmm.2016.2587

\begin{abstract}
The human zinc finger of the cerebellum (ZIC) family genes, comprised of 5 members, which are vertebrate homologues of the Drosophila odd-paired gene and encode zinc-finger transcription factors, have been shown to be involved in various diseases, including cancer. However, the roles of ZICs in human gastric cancer (GC) have not yet been fully elucidated. This study aimed to investigate the expression patterns of ZICs and determine their clinical significance in GC. The mRNA and protein expression levels of ZIC1-5 were detected by RT-qPCR and western blot analysis, respectively using 60 pairs of human GC and matched normal mucosa tissues. The expression pattern and subcellular localization of $\mathrm{ZIC1}$ in 160 pairs of human GC and matched normal mucosa tissues were verified by immunohistochemistry. Moreover, the associations of ZIC1 expression with various clinicopathological characteristics and patient prognosis were evaluated. The mRNA and protein expression levels of ZIC1 were both found to be significantly decreased in the GC tissues compared to matched normal mucosa tissues (GC vs. normal, 2.15 \pm 0.69 vs. 4.28 \pm 0.95 ; $\mathrm{P}<0.001)$; however, ZIC2-5 expression exhibited no significant difference between the cancer and normal tissue samples. In addition, the downregulation of ZIC1 (ZIC1-low) was more frequently observed in the GC tissues with positive lymph node metastasis $(\mathrm{P}=0.006)$, an advanced TNM stage $(\mathrm{P}<0.001)$ and a great depth of invasion $(\mathrm{P}=0.01)$. Notably, a low ZIC1 expression was significantly associated with a poor disease-free and overall survival. Furthermore, multivariate analysis revealed that ZIC1 expression was an independent prognostic marker for patients with GC. In conclusion, among the human ZIC family genes, the dysregulation of ZIC1, but not of ZIC2, ZIC3, ZIC4 and ZIC5, may play a crucial role in the progression of GC. ZIC1 may thus serve as a novel molecular marker to predict the progression, survival and relapse of patients with GC.
\end{abstract}

Correspondence to: Dr Xiaozhong Yang, Department of Gastroenterology, Huai'an First People's Hospital, Nanjing Medical University, 6 Beijing Road West, Huai'an, Jiangsu 223300, P.R. China E-mail: xzyangha@sina.com

Key words: gastric cancer, zinc finger of the cerebellum family genes, zinc finger protein of the cerebellum 1, clinicopathological characteristic, disease-free survival, overall survival

\section{Introduction}

Gastric cancer (GC), one of the most frequent malignancies, remains the second leading cause of cancer-related mortality worldwide (the third in males and the fifth in females), despite great advances in therapeutic regimens and improved surgical outcomes (1). Based on Globocan 2012, GC accounts for nearly 952,000 new cases annually in worldwide (2). Of all GC cases, $90 \%$ are malignant and $95 \%$ are comprised of gastric adenocarcinoma (3). More than $70 \%$ of new cases and deaths related to GC are occurred in developing countries (4). In China in particular, this cancer has the second highest incidence among commonly diagnosed cancers (5). Due to a lack of early specific symptoms, the diagnosis of GC is often delayed, leading to cancer cell invasion into the muscularis propria (6). Consistent with other types of cancer, the development of GC is a multiplestage process in which the accumulation of molecular changes lead to malignant phenotypes with aggressive characteristics (7). Therefore, the detection of the abnormal expression of molecular markers may be a promising approach for the early diagnosis and prognosis of patients with GC.

The human zinc finger of the cerebellum (ZIC) family genes, comprised of 5 members (ZIC1, ZIC2, ZIC3, ZIC4 and ZIC5) which are vertebrate homologues of the Drosophila odd-paired (OPA) gene, are structurally similar to each other, implying that ZICs share some, but not all, functions (8). ZIC genes encode zinc-finger transcription factors, each composed of $5 \mathrm{C}_{2} \mathrm{H}_{2}$ zinc-finger domains, which have highest sequence homology to Drosophila OPA (9). Functionally, ZIC genes play crucial roles in a wide array of developmental systems, including the central nervous system (CNS), muscle and skeletal development (10). The ZIC proteins are mainly expressed in the developing or mature CNS in a spatiotemporally restricted manner, but none contain a canonical nuclear localization signal. As zinc finger transcription factors, these proteins can bind to the GC-rich sequence in target genes (11). With the similar zinc finger domains, the ZIC family members have also been shown to interact with the Gli family proteins in both an antagonistic and synergistic manner (12). In recent years, growing evidence has indicated that ZICs may be involved in the pathological events of various diseases, including cancer. For example, ZIC1 was found to participate in the progression of human medulloblastoma (13), thyroid cancer (14), GC (15-17), colorectal cancer (18), endometrial cancer (19) and mesenchymal neoplasms (20); ZIC2 may function as an oncogene in small cell lung carcinoma (21), 
pancreatic ductal adenocarcinoma (22), epithelial ovarian cancer (23) and cervical cancer (24); genome-wide analysis of $\mathrm{CpG}$ island methylation in bladder cancer identified ZIC4 as a pTa-specific prognostic marker (25). However, the roles of the ZIC family members in GC have not yet been fully elucidated. A comparison of their expression levels and clinical significance in GC is required.

These observations led us to investigate the expression profiles of ZIC genes and proteins in GC, and to determine their clinical implications. We first detected the mRNA and protein expression levels of ZIC1, ZIC2, ZIC3, ZIC4 and ZIC5 by reverse transcription-quantitative polymerase chain reaction (RT-qPCR) and western blot analysis, respectively, using 60 pairs of human GC and matched normal mucosa tissues. The expression pattern and subcellular localization of ZIC1 in 160 pairs of human GC and matched normal mucosa tissues were then examined by immunohistochemistry. Moreover, the associations of ZIC1 expression with various clinicopathological characteristics and patient prognosis were also evaluated.

\section{Materials and methods}

Ethics statement. This study was approved by the Ethics Committee of Huai'an First People's Hospital of Nanjing Medical University and Lianshui Third People's Hospital, Huai'an, China. Written informed consent was also obtained from all study participants for the use of their samples. All specimens were handled anonymously according to the ethical and legal standards.

Patients and tissue samples. For RT-qPCR and western blot analysis, a total of 60 fresh GC and matched normal mucosa specimens were obtained from 60 patients with GC (48 males and 12 females; median age, 58 years; range, 28-82 years), who underwent surgical resection, at the Department of Gastroenterology of Huai'an First People's Hospital from January 2009 to December 2010. All specimens were stored at $-80^{\circ} \mathrm{C}$ until use to detect the relative expression levels of $\mathrm{ZIC1}$, ZIC2, ZIC3, ZIC4 and ZIC5 genes and proteins.

For immnohistochemistry, a total of 160 paraffin-embedded GC and matched normal mucosa specimens, in addition to the 60 cases mentioned above, were obtained from 160 patients with GC (108 males and 52 females; median age, 58 years; range, 28-86 years) at the Department of Gastroenterology of Huai'an First People's Hospital from January 2005 to December 2010.

None of the patients had received any radiotherapy or chemotherapy prior to surgery, and the samples were classified according to the World Health Organization (WHO) Pathological Classification of Tumors. Of the 160 cases, $58(36.25 \%)$ were well or moderately differentiated tumor tissues and $102(63.75 \%)$ were poorly differentiated or undifferentiated tumor tissues. Histologically, there were 10 cases of papillary adenocarcinoma, 92 cases of tubular adenocarcinoma, 50 cases of mucinous adenocarcinoma and 8 cases of signet-ring cell carcinoma. There were 61 cases of intestinal histological type and 99 cases of diffuse histological type according to the Lauren classification. According to the 2002 tumor-nodemetastases (TNM) classification system, 16 cases were TNM stage I, 40 cases were stage II, 42 cases were stage III and
62 cases were stage IV. The detailed information on the clinicopathological characteristics of all 160 patients with GC are shown in Table I.

All the 160 patients with GC were given a follow-up exam ranging from 3 to 6 years. Patients who died from diseases other than GC or from unexpected events were excluded from the case collection in this study. For the analysis of survival and follow-up data, the date of surgery was used to represent the beginning of the follow-up period. Overall survival was an endpoint which was calculated as the amount of time between the date of surgery and the date of death, regardless of the cause. Disease-free survival was defined as the time from randomization until recurrence of the tumor or death from any cause. Surviving patients were censored on March 31, 2013.

$R T-q P C R$. Total RNA was isolated using TRIzol reagent (Invitrogen, Carlsbad, CA, USA). A total of $2 \mu \mathrm{g}$ RNA was reverse transcribed using the SuperScript II RNase-Reverse Transcriptase system (Invitrogen). GAPDH was used as an internal control. cDNA was then subjected to quantitative (realtime) PCR (qPCR) using primers specific for ZIC1, ZIC2, ZIC3, ZIC4, ZIC5 and GAPDH. PCR primers were designed according to the previous study by Aruga et al (26) as follows: ZIC1 forward, 5'-GGCCCGGAGCAGAGTAAT-3' and reverse, 5'-AGCCCTCAAACTCGCACTT-3' (229 bp, 26 cycles); ZIC2 forward, 5'-CCCTTCAAGG CCAAATACAA-3' and reverse, 5'-TGCATGTGCTTCTTCCTGTC-3' (218 bp, 26 cycles); ZIC3 forward, 5'-GCAAGTCTTTCAAGGCGAAG-3' and reverse, 5'-CATGCATGTGCTTCTTACGG-3' (225 bp, 28 cycles); ZIC4 forward, 5'-GCCCTTCAAAGCCAAAT ACA-3' and reverse, 5'-GCCCTCGAACTCGCATC-3' (172 bp, 28 cycles); ZIC5 forward, 5'-TCTGCTTCTGGGAGGAC TGT-3' and reverse, 5'-GGGAATGTTTCTTCCGATCA-3' (252 bp, 28 cycles); and GAPDH forward, 5'-GAAGGTGAA GGTCGGAGT-3' and reverse, 5'-GAAGATGGTGATGGG ATTTC-3' (226 bp, 28 cycles). The PCR cycling conditions were as follows: $94^{\circ} \mathrm{C}$ for $4 \mathrm{~min}$, followed by 40 cycles of $95^{\circ} \mathrm{C}$ for $1 \mathrm{~min}, 60^{\circ} \mathrm{C}$ for $1 \mathrm{~min}$ and $72^{\circ} \mathrm{C}$ for $1 \mathrm{~min}$. The SYBR Premix Ex Taq ${ }^{\mathrm{TM}}$ kit (Takara Bio, Inc., Otsu, Shiga, Japan) was used to measure the amplified DNA, and qPCR was performed using an iQ5 real-time PCR detection system (Bio-Rad, Hercules, CA, USA). The amount of ZICs relative to GAPDH was calculated as the average $2^{-\Delta \mathrm{Ct}}$, where $\Delta \mathrm{Ct}=\mathrm{Ct}-\mathrm{Ct}_{\mathrm{GAPDH}}$.

Western blot analysis. The GC and matched normal mucosa tissues were added to $1 \mathrm{ml}$ of $100 \mathrm{mmol} / \mathrm{l}$ Tris- $\mathrm{HCl}(\mathrm{pH} \mathrm{7.5)}$, $100 \mathrm{mmol} / 1 \mathrm{NaCl}, 0.5 \%$ sodium deoxycholate, $1 \mathrm{mmol} / \mathrm{l}$ ethylenediaminetetraacetic acid, $1 \%$ Nonidet P-40, and $0.1 \%$ sodium dodecyl sulfate and protease inhibitor. Lysates $(100 \mu \mathrm{g})$ were resolved on an SDS-PAGE gel and transferred to PVDF membranes (Millipore, Bedford, MA, USA). After blocking in 5\% milk with TBST, the memberanes were inclubated with ZIC1 (1:500, ab134951), ZIC2 (1:500, ab150404), ZIC3 (1:500, ab136431), ZIC4 (1:500, ab199284), ZIC5 (1:500, ab42483) and GAPDH (1:1000, ab181602) (all from Abcam, Cambridge, UK) antibodies at room temperature overnight. Subsequently, secondary antibodies coupled to horseradish peroxidase (HRP) were visualized using a chemiluminescence with Las-4000 Imaging system (Fujifilm, Tokyo, Japan). The relative expression levels of proteins were quantified and normalized to GAPDH. 
Table I. Associations of ZIC1 expression with various clinicopathological characteristics of the 160 patients with GC.

\begin{tabular}{|c|c|c|c|c|}
\hline \multirow[b]{2}{*}{ Clinical characteristics } & \multirow[b]{2}{*}{ Case no. $(\%)$} & \multicolumn{2}{|c|}{ ZIC1 expression } & \multirow[b]{2}{*}{ P-value } \\
\hline & & $\operatorname{High}(\mathrm{n}, \%)$ & Low (n, \%) & \\
\hline \multicolumn{5}{|l|}{ Age (years) } \\
\hline$<58$ & $50(31.25)$ & $25(50.00)$ & $25(50.00)$ & \multirow[t]{2}{*}{ NS } \\
\hline$\geq 58$ & $110(68.75)$ & $50(45.45)$ & $60(54.55)$ & \\
\hline \multicolumn{5}{|l|}{ Gender } \\
\hline Male & $108(67.50)$ & $52(48.15)$ & $56(51.85)$ & \multirow[t]{2}{*}{ NS } \\
\hline Female & $52(32.50)$ & $23(44.23)$ & $29(55.77)$ & \\
\hline \multicolumn{5}{|l|}{ Tumor size $(\mathrm{cm})$} \\
\hline$<4$ & $58(36.25)$ & $25(43.10)$ & $33(56.90)$ & \multirow[t]{2}{*}{ NS } \\
\hline$\geq 4$ & $102(63.75)$ & $50(49.02)$ & $52(50.98)$ & \\
\hline \multicolumn{5}{|l|}{ Lauren classification } \\
\hline Diffuse type & $99(61.88)$ & $45(45.45)$ & $54(54.55)$ & \multirow{2}{*}{ NS } \\
\hline Intestinal type & $61(38.12)$ & $30(49.18)$ & $31(50.82)$ & \\
\hline \multicolumn{5}{|l|}{ Differentiation } \\
\hline Well or moderate & $58(36.25)$ & $29(50.00)$ & $29(50.00)$ & \multirow[t]{2}{*}{ NS } \\
\hline Poor & $102(63.75)$ & $46(45.10)$ & $56(54.90)$ & \\
\hline \multicolumn{5}{|l|}{ Lymph node metastasis } \\
\hline Negative & $100(62.50)$ & $60(60.00)$ & $40(40.00)$ & \multirow[t]{2}{*}{0.006} \\
\hline Positive & $60(37.50)$ & $15(25.00)$ & $45(75.00)$ & \\
\hline \multicolumn{5}{|l|}{ TNM stage } \\
\hline I & $16(10.00)$ & $16(100.00)$ & $0(0.00)$ & \multirow[t]{4}{*}{$<0.001$} \\
\hline II & $40(25.00)$ & $37(92.50)$ & $3(7.50)$ & \\
\hline III & $42(26.25)$ & $22(52.38)$ & $20(47.62)$ & \\
\hline IV & $62(38.75)$ & $0(0.00)$ & $62(100.00)$ & \\
\hline \multicolumn{5}{|l|}{ Depth of invasion } \\
\hline Mucosa or submucosa & $26(16.25)$ & $24(92.31)$ & $2(7.69)$ & \multirow{4}{*}{0.01} \\
\hline Muscularis or subserosa & $20(12.50)$ & $12(60.00)$ & $8(40.00)$ & \\
\hline Serosa & $82(51.25)$ & $37(45.12)$ & $45(54.88)$ & \\
\hline Adjacent structure & $32(20.00)$ & $2(6.25)$ & $30(93.75)$ & \\
\hline
\end{tabular}

NS, refers to the difference without statistical significance; ZIC1, zinc finger of the cerebellum 1; GC, gastric cancer; TNM, tumor-node-metastasis .

Immunohistochemistry. The subcellular localization and expression pattern of ZIC1 protein were examined by immunohistochemistry using $4-\mu \mathrm{m}$-thick formalin-fixed paraffin embedded sections of GC and matched normal mucosa tissues. The tissue sections were dewaxed in xylene, rehydrated in alcohol, immersed in 3\% hydrogen peroxide for $10 \mathrm{~min}$, and the slides were then treated with antigen in $0.01 \mathrm{~mol} / \mathrm{l}$ sodium citrate buffer in a microwave for $20 \mathrm{~min}$ before incubating overnight at $4^{\circ} \mathrm{C}$ with ZIC1 primary antibody [ZIC1 (1:500, ab134951; Abcam)] in 3\% bovine serum albumin (BSA). Subsequently, the sections were washed in $50 \mathrm{mM}$ Tris buffer at pH 7.6 3 times, and the biotinylated link antibody (Dako Corp., Carpinteria, CA, USA) and streptavidin-peroxidase conjugate were applied sequentially for $15 \mathrm{~min}$ each. The Dako liquid 3,3'-diaminobenzidine (DAB) substrate-chromogen solution was used as the chromogen, and hematoxylin was used as the nuclear counterstain. Finally, the tissue sections were counterstained with hemalum, before being dehydrated, cleared and mounted. Non-specific binding was assessed using a non-immune rabbit serum $(1: 1,000)$ in $3 \%$ BSA in the place of the primary antibody.

To evaluate the results of immunohistochemical staining, the stained sections were reviewed by 2 independent pathologists blinded the clinicopathological characteristics of the patients with GC. They randomly selected 5 visual fields for each section and counted the number of total tumor cells and positive cells, and recorded the staining intensity. The semi-quantitative method was used to determine the staining intensity of the samples according to the staining intensity and the percentage of positively stained cells. The staining intensity was classified into 4 grades: 0 , none; 1 , poor; 2 , moderate and 3 , strong. The percentage of positive cells $(\mathrm{P})$ was scored as follows: $0,0 \% ; 1,1-25 \% ; 2,26-50 \% ; 3,50-75 \%$; and $4,>75 \%$. The total immunoreactive score (IRS) was calculated as the 
A
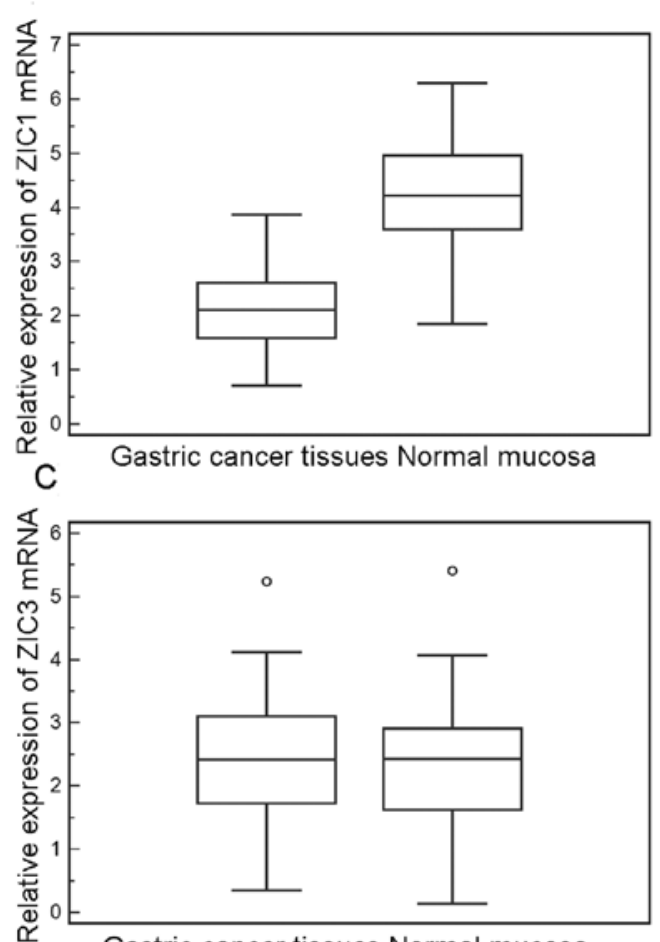

Gastric cancer tissues Normal mucosa
B

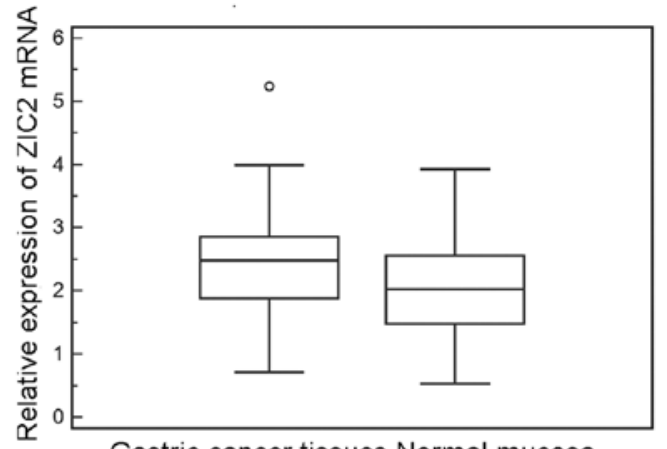

Dastric cancer tissues Normal mucosa

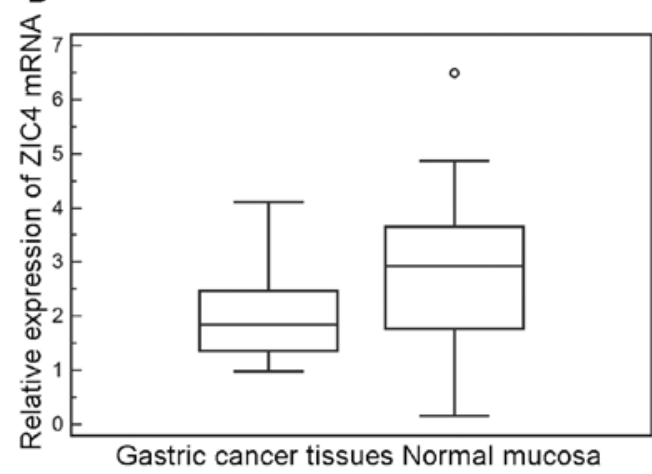

E

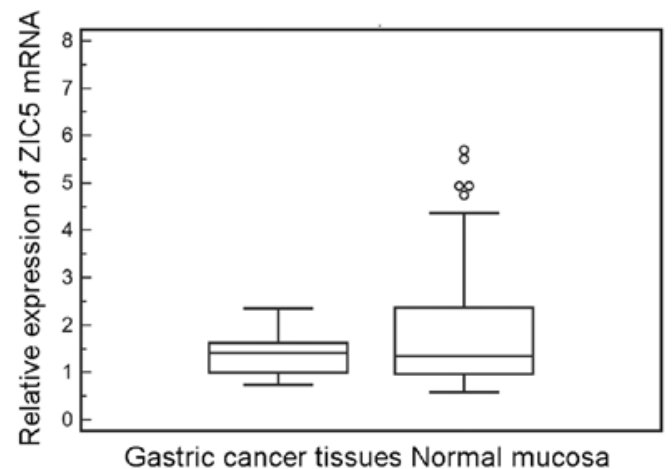

Figure 1. Relative mRNA expression of zinc finger of the cerebellum (ZIC) genes. (A) ZIC1, (B) ZIC2, (C) ZIC3, (D) ZIC4 and (E) ZIC5 in gastric cancer (GC) and matched normal mucosa tissue samples.

staining intensity score multiplied by the percentage of positively stained cells. IRS $\leq 2.09$ (median value of IRS in GC tissues) was defined as a low expression, and IRS $>2.09$ was defined as a high expression.

Statistical analysis. All statistical analyses were performed using SPSS software version 11.0 for Windows (SPSS, Inc., Chicago, IL, USA). Continuous variables were expressed as the means $\pm \mathrm{SD}$. The differences of ZIC expression, at the mRNA and protein level, between the GC tissues and matched normal mucosa were analyzed by an paired-t test. The associations of ZIC1 expression with various clinicopathological characteristics of the patients with GC were analyzed by Fisher's exact test for any $2 \times 2$ tables and Pearson's $\chi^{2}$ test for non- $2 \times 2$ tables. The survival analysis was estimated using the Kaplan-Meier method and data were compared using the log-rank test. Multivariate analysis was performed using the Cox proportional hazard model. A value of $\mathrm{P}<0.05$ was considered to indicate a statistically significant difference.

\section{Results}

Expression of ZICl-5 in human GC tissues. We first detected the expression of the 5 ZIC genes (ZIC1-5) in 60 fresh GC tissue and matched normal mucosa samples by RT-qPCR. As shown in Fig. 1, the relative mRNA expression level of ZIC1 was significantly decreased in the GC tissues compared to the matched normal mucosa tissues (GC vs. normal, 2.15 \pm 0.69 vs. $4.28 \pm 0.95 ; \mathrm{P}<0.001)$; however, the expression levels of ZIC2 (GC vs. normal, $2.42 \pm 0.84$ vs. $2.08 \pm 0.74$; $\mathrm{P}>0.05$ ), ZIC3 (GC vs. normal, $2.28 \pm 1.13$ vs. $2.24 \pm 1.13, \mathrm{P}>0.05$ ), ZIC4 (GC vs. normal, $2.07 \pm 0.86$ vs. $2.63 \pm 1.46, \mathrm{P}>0.05$ ) and ZIC5 (GC vs. normal, $1.38 \pm 0.39$ vs. $1.98 \pm 1.53, \mathrm{P}>0.05$ ) exhibited no significant differences between the cancer and normal tissue samples, in line with the findings of western blot analysis (Fig. 2). Notably, in the 60 fresh normal mucosa samples, ZIC1 mRNA and protein levels were 1.02- to 5.35fold and 1.5- to 3.2-fold higher, respectively, than those in the GC tissues. 

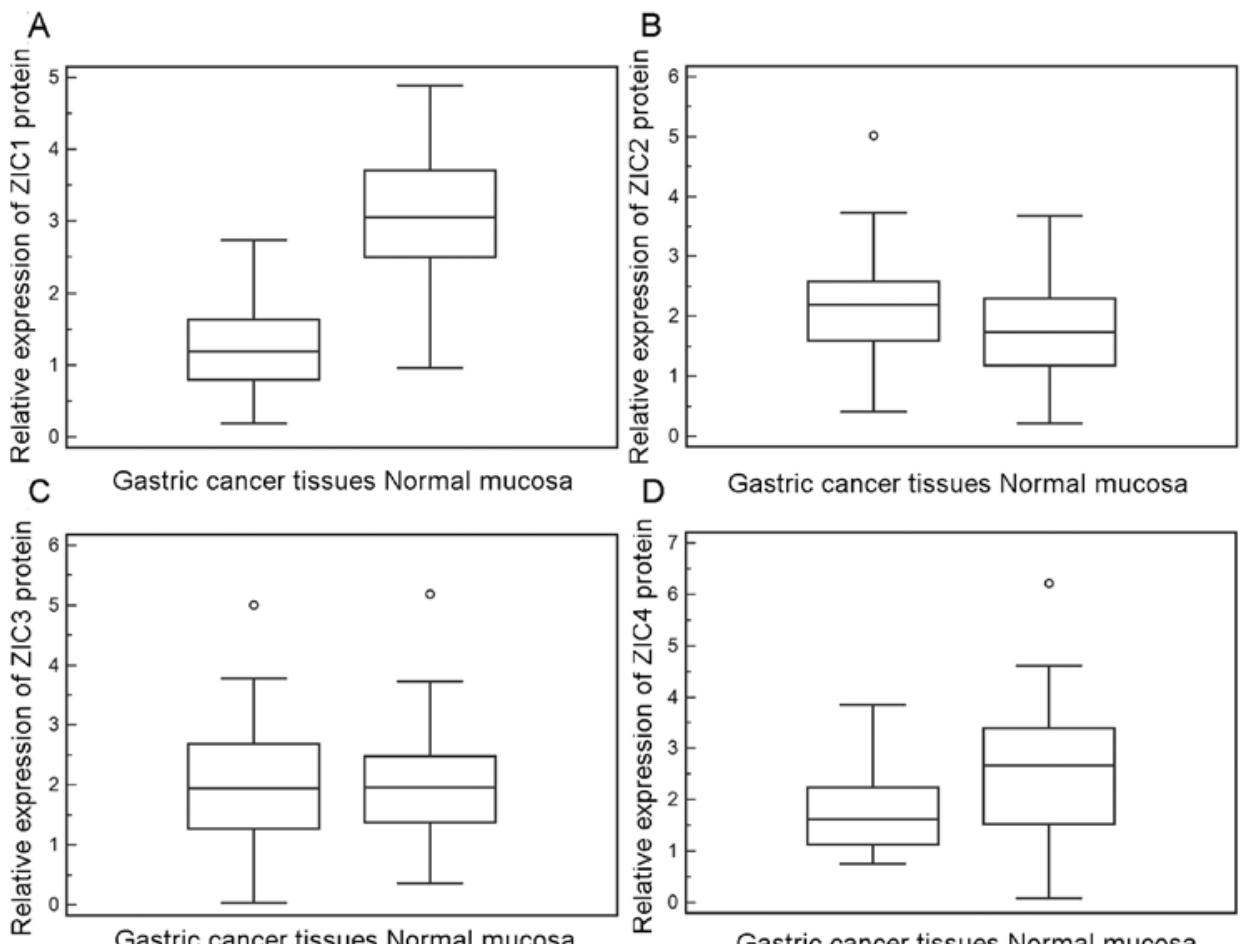

D Gastric cancer tissues Normal mucosa
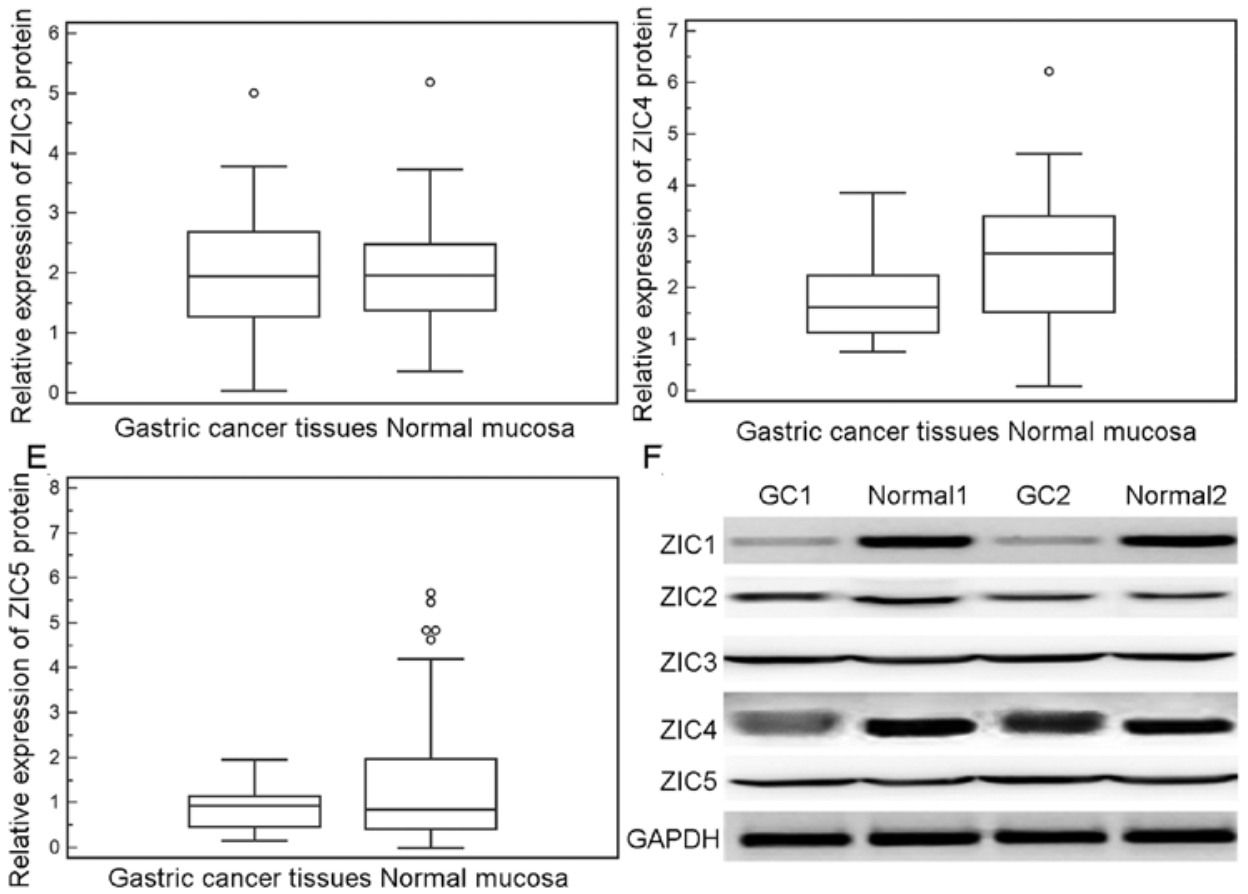

Figure 2. Relative protein expression of zinc finger of the cerebellum (ZIC) 1 genes.(A) ZIC1, (B) ZIC2, (C) ZIC3, (D) ZIC4 and (E) ZIC5 in gastric cancer (GC) and matched normal mucosa. (F) Represent blots of these proteins.

Moreover, immunohistochemistry revealed that the positive staining for $\mathrm{ZIC1}$ protein in the GC tissues and matched normal mucosa tissue samples was predominantly localized in the cell cytoplasm (Fig. 3A). There was a significant difference in $\mathrm{ZICl}$ expression between the GC and matched normal mucosa samples from the same patient (GC vs. normal, 2.33 \pm 0.81 vs. $4.20 \pm 1.09, \mathrm{P}<0.001$; Fig. $3 \mathrm{C}$ ).

Downregulation of ZICl is associated with the aggressive progression of human GC. To assess the association between ZIC1 expression and various clinicopathological characteristics, the median value (2.09) of ZIC1 expression in GC tissues was used as a cut-off point for dividing all $160 \mathrm{GC}$ patients into the ZIC1-low and ZIC1-high groups. GC patients with an IRS of ZIC1 exceeding the median value were deemed to be in the ZIC1-high group; all other tissues were considered to be in the ZIC1-low group. Of the 160 GC patients, 75 (46.88\%) displayed a high expression of ZIC1 and 85 (53.12\%) exhibited a low expression of ZIC1. As shown in Table I, the downregulation of ZIC1 (ZIC1-low) was more frequently observed in the GC tissues with positive lymph node metastasis $(\mathrm{P}=0.006)$, an advanced TNM stage $(\mathrm{P}<0.001)$ and a great depth of invasion $(\mathrm{P}=0.01)$. However, there was no significant relation observed between ZIC1 and other clinicopathological characteristics such as age, gender, tumor size, lauren classification, and tumor differentiation (all $\mathrm{P}>0.05$; Table I).

Downregulation of ZIC1 is associated with a poor prognosis in human GC. Kaplan-Meier analysis revealed that the GC patients with a low ZIC1 expression had both a shorter overall survival and disease-free survival than those with a high ZIC1 expression (both $\mathrm{P}=0.001$, log-rank test; Fig. 4). To determine whether ZIC1 expression is an independent risk factor for prognosis, the Cox proportional hazard regression model was used. As shown in Table II, univariate analysis revealed that positive lymph node metastasis (both $\mathrm{P}=0.01$ ), an advanced TNM stage (both $\mathrm{P}<0.001$ ), a great depth of invasion ( $\mathrm{P}=0.02$ and 0.03 , respectively) and a low $\mathrm{ZIC1}$ expression (both $\mathrm{P}=0.001$ ) were significantly associated with a poor overall survival and disease-free survival. Furthermore, multivariate analysis revealed that the status of lymph node metastasis [for overall survival, hazard ratio $(\mathrm{HR})=4.658$, 


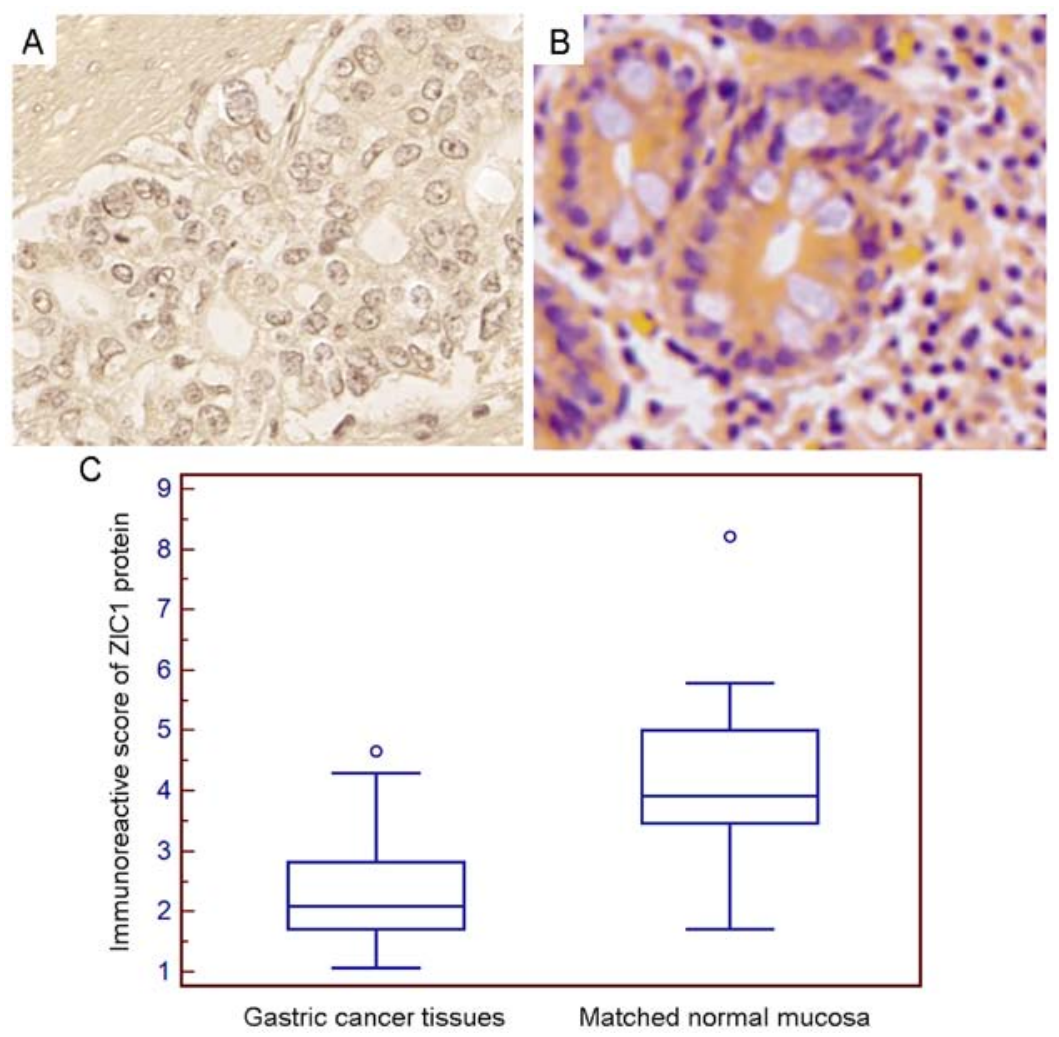

Figure 3. Validation of differentially expressed zinc finger of the cerebellum 1 (ZIC1) by immunohistochemistry. Immunohistochemistry revealed the positive staining of ZIC1 protein in (A) gastric cancer (GC) tissues and (B) matched normal mucosa. Staining was predominantly observed in the cell cytoplasm (x200 magnification). (C) There was a significant difference in ZIC1 expression between GC and matched normal mucosa tissues from the same patient (GC vs. normal, $2.33 \pm 0.81$ vs. $4.20 \pm 1.09, \mathrm{P}<0.001)$.
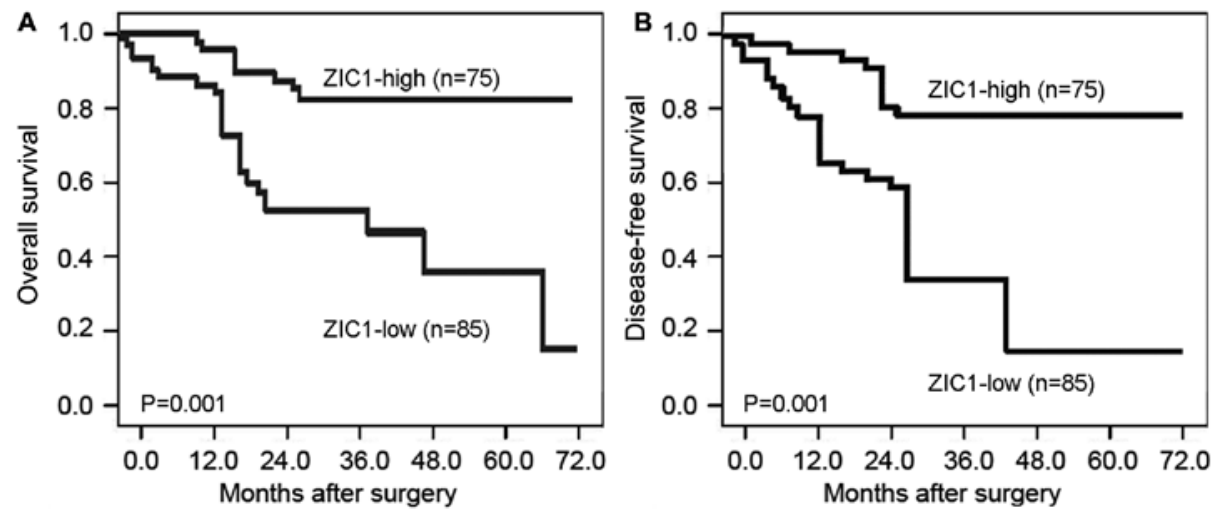

Figure 4. Kaplan-Meier survival curves of (A) overall survival and (B) disease-free survival for zinc finger of the cerebellum 1 (ZIC1) expression in human gastric cancer (GC).

95\% $\mathrm{CI}=0.913-9.621, \mathrm{P}=0.01$; for disease-free survival, $\mathrm{HR}=4.126,95 \% \mathrm{CI}=0.893-9.282, \mathrm{P}=0.01]$, TNM stage (for overall survival, $\mathrm{HR}=10.039,95 \% \mathrm{CI}=1.925-20.791, \mathrm{P}<0.001$; for disease-free survival, $\mathrm{HR}=9.629,95 \% \mathrm{CI}=1.902-19.391$, $\mathrm{P}<0.001$ ), depth of invasion (for overall survival, $\mathrm{HR}=3.939$, 95\% $\mathrm{CI}=0.825-8.791, \mathrm{P}=0.02$; for disease-free survival, $\mathrm{HR}=3.027,95 \% \mathrm{CI}=0.628-7.128, \mathrm{P}=0.03$ ) and $\mathrm{ZIC1}$ expression (for overall survival, $\mathrm{HR}=4.928,95 \% \mathrm{CI}=0.936-10.362, \mathrm{P}=0.01$; for disease-free survival, $\mathrm{HR}=4.068,95 \% \mathrm{CI}=0.882-8.968$, $\mathrm{P}=0.02$ ) were independent prognostic markers for predicting poor prognosis in patients with GC.

\section{Discussion}

Human GC represents a devastating disease without early symptoms, and it is associated with a rapid progression and poor prognosis, leading to an increasing mortality rate. Thus, it is extremely necessary to identify novel and efficient biomarkers for the early diagnosis and prognosis of this cancer. In the present study, we detected the mRNA and protein expression levels of ZIC1, ZIC2, ZIC3, ZIC4 and ZIC5 in human GC tissues and adjacent normal mucosa tissue sampoles, but found that only ZIC1 had a differential expression between the cancer 
Table II. Prognostic value of ZIC1 expression for the overall survival and disease-free survival of patients with GC by multivariate analysis with Cox regression.

\begin{tabular}{|c|c|c|c|c|}
\hline \multirow[b]{2}{*}{ Characteristics } & \multicolumn{2}{|c|}{ Overall survival } & \multicolumn{2}{|c|}{ Disease-free survival } \\
\hline & $\mathrm{HR}(95 \% \mathrm{CI})$ & P-value & HR $(95 \% \mathrm{CI})$ & P-value \\
\hline Age & $1.005(0.101-2.008)$ & NS & $0.798(0.122-1.689)$ & NS \\
\hline Gender & $1.379(0.303-2.743)$ & NS & $1.199(0.336-2.306)$ & NS \\
\hline Tumor size & $0.462(0.202-1.039)$ & NS & $0.729(0.308-1.692)$ & NS \\
\hline Lauren classification & $2.232(0.566-4.676)$ & NS & $1.928(0.416-4.086)$ & NS \\
\hline Differentiation & $1.019(0.272-2.069)$ & NS & $1.012(0.262-2.016)$ & NS \\
\hline Lymph node metastasis & $4.658(0.913-9.621)$ & 0.01 & $4.126(0.893-9.282)$ & 0.01 \\
\hline TNM stage & $10.039(1.925-20.791)$ & $<0.001$ & $9.629(1.902-19.391)$ & $<0.001$ \\
\hline Depth of invasion & $3.939(0.825-8.791)$ & 0.02 & $3.027(0.628-7.128)$ & 0.03 \\
\hline ZIC1 expression & $4.928(0.936-10.362)$ & 0.01 & $4.068(0.882-8.968)$ & 0.02 \\
\hline
\end{tabular}

ZIC1, zinc finger of the cerebellum 1; GC, gastric cancer; TNM, tumor-node-metastasis; HR, hazard ratio.

and normal tissues. In addition, our immunohistochemical analysis verified the downregulation of ZIC1 protein in GC tissues, and showed that it significantly correlated with lymph node metastasis, a higher TNM stage and a deeper invasion. Moreover, Kaplan-Meier curve analysis demonstrated that the GC patients with a low ZIC1 expression had a poorer prognosis than those with a high ZIC1 expression. ZIC1 expression was identified as an independent factor of the overall and diseasefree survivals in GC patients by multivariate Cox analysis. These results suggested that $\mathrm{ZIC1}$ may serve as a biomarker to predict the progression and prognosis of GC patients.

As one of the $5 \mathrm{ZIC}$ family genes, $\mathrm{ZIC1}$ is located on chromosome $3 \mathrm{q} 24$ and encodes a $\mathrm{C}_{2} \mathrm{H}_{2}$-type zinc finger transcription factor which is noted for its involvement in various biological processes, including early patterning, neurogenesis, dorsal neural tube development, myogenesis and left-right axis establishment by regulating cell growth, proliferation and differentiation (27). Under physiological conditions, the expression of ZIC1 has only been found in neural tissues, and is restricted to the cerebellum (28). In recent years, growing evidence has indicatd that ZIC1 may play a critical role in the progression of several types of cancer, due to its involvement in fibroproliferative processes and its regulatory effects on several members of the Wnt and Notch signaling pathways (29). Gan et al (18) reported that $\mathrm{ZIC1}$ expression was markedly downregulated in primary colorectal cancer tissues as compated to adjacent non-tumor tissues through promoter hypermethylation, and acted as a tumor suppressor to inhibit cell proliferation and to induce apoptosis by interacting with the MAPK and $\mathrm{PI} 3 \mathrm{~K} / \mathrm{Akt}$ pathways, as well as the $\mathrm{Bcl}-\mathrm{xl} / \mathrm{Bad} / \mathrm{caspase}-3$ cascade. Qiang et al (14) indicated that ZIC1 was frequently downregulated by promoter hypermethylation in both primary thyroid cancer tissues and thyroid cancer cell lines, and demonstrated that ZIC1 hypermethylation was significantly associated with lymph node metastasis in patients with papillary thyroid cancer, and was also identified as a putative tumor suppressor by modulating major signaling pathways and the transcription factor FOXO3a. By contrast, Brill et al (30) revealed that ZIC1 was overexpressed in all 5 subtypes of liposarcoma compared with normal fat and in liposarcoma cell lines compared with adipose-derived stem cells, and may function as an oncogene to promote proliferation and invasion, and to suppress apoptosis in dedifferentiated and myxoid/round cell liposarcoma cell lines. These previous findings suggest that ZIC1 may contribute to cancer progression in a cancer-specific manner.

As regards GC, Wang et al (15) in 2009 firstly found that ZIC1 expression was distinctly decreased in primary GC tissues in comparison with non-tumor adjacent gastric tissues through promoter hypermethylation, and revealed that the ectopic expression of ZIC1 led to the growth inhibition of GC cells through the induction of S-phase cell cycle arrest; In 2012, the same research group indicated that the overexpression of ZIC1 could result in the inactivation of the Shh, PI3K and MAPK signaling pathways, as well as in the regulation of multiple downstream targets which may be essential for the development and progression of GC (16); In 2015, Chen et al (17) also identified ZIC1 promoter hypermethylation in plasma DNA as a potential biomarker for GC and intraepithelial neoplasia. Consistently, our data verified the downregulation of ZIC1, at both the mRNA and protein level in GC tissues, by RT-qPCR, western blot analysis and immunohistochemistry, respectively. In addition, the ZIC1 expression data obtained from immunohistochemical detection were analyzed for correlations with clinicopathological characteristics. As a result, we found that a low ZIC1 expression significantly correlated with lymph node metastasis, a deeper invasion, and a higher TNM stage, suggesting that ZIC1 downregulation may affect the invasion, metastasis and progression of GC, and may indicate the aggressive behavior of cancer. Apart from the results mentioned above, Kaplan-Meier curves proved that the patients with a low ZIC1 expression presented with poorer overall and diseasefree survivals than the patients with a high ZIC1 expression. Multivariate Cox regression analysis confirmed that ZIC1 expression, along with lymph node metastasis, TNM stage and depth of invasion, was an independent prognostic factor of GC prognosis. To the best of our knowledge, this is first study to confirm the clinical significance of ZIC1 in GC based on a large cohort of clinical samples. 
In conclusion, among the human ZIC family genes, ZIC1 dysregulation, but not that of ZIC2, ZIC3, ZIC4 and ZIC5, may play a crucial role in GC progression. ZIC1 may serve as a novel molecular marker to predict the progression, survival and relapse of patients with GC.

\section{References}

1. Torre LA, Bray F, Siegel RL, Ferlay J, Lortet-Tieulent J and Jemal A: Global cancer statistics, 2012. CA Cancer J Clin 65: 87-108, 2015

2. Colquhoun A, Arnold M, Ferlay J, Goodman KJ, Forman D and Soerjomataram I: Global patterns of cardia and non-cardia gastric cancer incidence in 2012. Gut 64: 1881-1888, 2015.

3. Qian X, Hu J, Zhao J and Chen H: ATP citrate lyase expression is associated with advanced stage and prognosis in gastric adenocarcinoma. Int J Clin Exp Med 8: 7855-7860, 2015.

4. Rahman R, Asombang AW and Ibdah JA: Characteristics of gastric cancer in Asia. World J Gastroenterol 20: 4483-4490, 2014.

5. He ZX and Li B: Recent progress in genetic and epigenetic profile of diffuse gastric cancer. Cancer Transl Med 1: 80-93, 2015.

6. Hashimoto T, Arai K, Yamashita Y, Iwasaki Y and Hishima T: Characteristics of intramural metastasis in gastric cancer. Gastric Cancer 16: 537-542, 2013.

7. Kanda $\mathrm{M}$ and Kodera Y: Recent advances in the molecular diagnostics of gastric cancer. World J Gastroenterol 21: 9838-9852, 2015.

8. Ali RG, Bellchambers HM and Arkell RM: Zinc fingers of the cerebellum (Zic): Transcription factors and co-factors. Int $\mathrm{J}$ Biochem Cell Biol 44: 2065-2068, 2012.

9. Houtmeyers R, Souopgui J, Tejpar S and Arkell R: The ZIC gene family encodes multi-functional proteins essential for patterning and morphogenesis. Cell Mol Life Sci 70: 3791-3811, 2013.

10. Bataller L, Wade DF, Fuller GN, Rosenfeld MR and Dalmau J: Cerebellar degeneration and autoimmunity to zinc-finger proteins of the cerebellum. Neurology 59: 1985-1987, 2002.

11. Sakurai H, Kikuchi K, Tsuchiya T, Kanazawa $H$ and Tsuda $M$ : Developmentally and regionally regulated alterations of octamerand GC-box-binding activities during the postnatal development of mouse cerebellum. Brain Res Dev Brain Res 61: 161-168, 1991.

12. Aruga J, Nagai T, Tokuyama T, Hayashizaki Y, Okazaki Y, Chapman VM and Mikoshiba K: The mouse zic gene family. Homologues of the Drosophila pair-rule gene odd-paired. J Biol Chem 271: 1043-1047, 1996.

13. Lacroix J, Schlund F, Leuchs B, Adolph K, Sturm D, Bender S, Hielscher T, Pfister SM, Witt O, Rommelaere J, et al: Oncolytic effects of parvovirus H-1 in medulloblastoma are associated with repression of master regulators of early neurogenesis. Int $\mathrm{J}$ Cancer 134: 703-716, 2014

14. Qiang W, Zhao Y, Yang Q, Liu W, Guan H, Lv S, Ji M, Shi B and Hou P: ZIC1 is a putative tumor suppressor in thyroid cancer by modulating major signaling pathways and transcription factor FOXO3a. J Clin Endocrinol Metab 99: E1163-E1172, 2014.

15. Wang LJ, Jin HC, Wang X, Lam EK, Zhang JB, Liu X, Chan FK, Si JM and Sung JJ: ZIC1 is downregulated through promoter hypermethylation in gastric cancer. Biochem Biophys Res Commun 379: 959-963, 2009.
16. Zhong J, Chen S, Xue M, Du Q, Cai J, Jin H, Si J and Wang L: ZIC1 modulates cell-cycle distributions and cell migration through regulation of sonic hedgehog, PI(3)K and MAPK signaling pathways in gastric cancer. BMC Cancer 12: 290, 2012.

17. Chen X, Lin Z, Xue M, Si J and Chen S: Zicl promoter hypermethylation in plasma DNA is a potential biomarker for gastric cancer and intraepithelial neoplasia. PLoS One 10: e0133906, 2015.

18. Gan L, Chen S, Zhong J, Wang X, Lam EK, Liu X, Zhang J, Zhou T, Yu J, Si J, et al: ZIC1 is downregulated through promoter hypermethylation, and functions as a tumor suppressor gene in colorectal cancer. PLoS One 6: e16916, 2011.

19. Wong YF, Cheung TH, Lo KW, Yim SF, Siu NS, Chan SC, Ho TW, Wong KW, Yu MY, Wang VW, et al: Identification of molecular markers and signaling pathway in endometrial cancer in Hong Kong Chinese women by genome-wide gene expression profiling. Oncogene 26: 1971-1982, 2007.

20. Pourebrahim R, Van Dam K, Bauters M, De Wever I, Sciot R, Cassiman JJ and Tejpar S: ZIC1 gene expression is controlled by DNA and histone methylation in mesenchymal proliferations. FEBS Lett 581: 5122-5126, 2007.

21. Vural B, Chen LC, Saip P, Chen YT, Ustuner Z, Gonen M, Simpson AJ, Old LJ, Ozbek U and Gure AO: Frequency of SOX Group B (SOX1, 2, 3) and ZIC2 antibodies in Turkish patients with small cell lung carcinoma and their correlation with clinical parameters. Cancer 103: 2575-2583, 2005.

22. Inaguma $\mathrm{S}$, Ito $\mathrm{H}$, Riku $\mathrm{M}$, Ikeda $\mathrm{H}$ and Kasai $\mathrm{K}$ : Addiction of pancreatic cancer cells to zinc-finger transcription factor ZIC2. Oncotarget 6: 28257-28268, 2015.

23. Marchini S, Poynor E, Barakat RR, Clivio L, Cinquini M, Fruscio R, Porcu L, Bussani C, D'Incalci M, Erba E, et al: The zinc finger gene ZIC2 has features of an oncogene and its overexpression correlates strongly with the clinical course of epithelial ovarian cancer. Clin Cancer Res 18: 4313-4324, 2012.

24. Chan DW, Liu VW, Leung LY, Yao KM, Chan KK, Cheung AN and Ngan HY: Zic2 synergistically enhances hedgehog signalling through nuclear retention of Gli1 in cervical cancer cells. J Pathol 225: 525-534, 2011.

25. Kandimalla R, van Tilborg AA, Kompier LC, Stumpel DJ, Stam RW, Bangma CH and Zwarthoff EC: Genome-wide analysis of $\mathrm{CpG}$ island methylation in bladder cancer identified TBX2, TBX3, GATA2, and ZIC4 as pTa-specific prognostic markers. Eur Urol 61: 1245-1256, 2012.

26. Aruga J, Nozaki Y, Hatayama M, Odaka YS and Yokota N: Expression of ZIC family genes in meningiomas and other brain tumors. BMC Cancer 10: 79, 2010.

27. Degreef I, De Smet L, Sciot R, Cassiman JJ and Tejpar S: Immunohistochemical evidence for Zic1 coexpression with beta-catenin in the myofibroblast of Dupuytren disease. Scand J Plast Reconstr Surg Hand Surg 43: 36-40, 2009.

28. Sabater L, Bataller L, Suárez-Calvet M, Saiz A, Dalmau J and Graus F: ZIC antibodies in paraneoplastic cerebellar degeneration and small cell lung cancer. J Neuroimmunol 201-202: 163-165, 2008

29. Merzdorf CS: Emerging roles for zic genes in early development. Dev Dyn 236: 922-940, 2007.

30. Brill E, Gobble R, Angeles C, Lagos-Quintana M, Crago A, Laxa B, Decarolis P, Zhang L, Antonescu C, Socci ND, et al: ZIC1 overexpression is oncogenic in liposarcoma. Cancer Res 70: 6891-6901, 2010. 